\section{Laudatio zur Emeritierung von Professor Dr. med. Hans Heinz Schild}

Professor Dr. med. Hans Heinz Schild wurde 1953 in der Stadt Remagen bei Bonn geboren. Nach seinem Abitur am Staatlichen-Nicolaus-Cusanus-Gymnasium in Bad Godesberg absolvierte er sein Medizinstudium an der Rheinischen-FriedrichWilhelms-Universität Bonn, welches er 1976 abschloss. Zeitgleich legte er das amerikanische Examen der Educational Commission for Foreign Medical Graduates erfolgreich ab. Die radiologische Ausbildung absolvierte er an der Klinik mit Poliklinik für Radiologie der Universität Mainz, unterbrochen durch einen längeren Gastarztaufenthalt am Department of Radiology der University of California San Francisco.

Die Ernennung zum Oberarzt erfolgte 1985 an der Radiologischen Universitätsklinik Mainz, die Venia Legendi für das Fachgebiet Radiologie erhielt er im selben Jahr. Im folgenden Jahr erfolgte in Mainz die Ernennung zum Universitätsprofessor (C2) auf Lebenszeit. Ende 1993 wurde Professor Schild auf die C4-Professur für Radiologie an die Rheinische-Friedrich-Wilhelms-Universität Bonn berufen. Mit Antritt der Stelle als Direktor leitete er seit 1994 in der Radiologischen Klinik auch die Neuroradiologie und die Strahlentherapie.

Neben seiner Tätigkeit als Klinikdirektor und seinem Engagement in der akademischen Forschung und Lehre ist Professor Schild seit vielen Jahren Mitherausgeber der Röfo und Radiologie up2date und war Mitglied im Editorial Board sowie Consultant to the Editor der Zeitschrift Radiology. Die klinischen und wissenschaftlichen Schwerpunkte von Prof. Schild liegen neben der Hochfeld-MRT insbesondere auf dem Gebiet der interventionellen Radiologie.

Seiner Initiative ist es zu verdanken, dass Bonn einer der ersten Standorte weltweit war, an denen ein 3Tesla-Hochfeld-MRT in den klinischen Betrieb ging. Auf der Basis der klinischen Erfahrungen und wissenschaftlichen Projekte auf dem Gebiet der Hochfeld-MRT wurde in Bonn über 10 Jahre ein „International Symposium on Highfield MR in Clinical Applications“ ausgerichtet, welches großen internationalen Zuspruch fand und Wissenschaftler aus über 13 verschiedenen Nationen nach Bonn führte.

Mit dem ersten HIFU-Gerät im deutschsprachigen Raum nahm die Bonner Radiologie auch im Bereich der interventionelIen Radiologie eine Vorreiterrolle wahr. Bereits seit 2014 werden dort mittels hochfokussiertem Ultraschall unterschiedliche Tumore gezielt und nicht invasiv behandelt. Zudem sind sein besonderes Engagement und die Weiterentwicklung von Diagnostik und Therapieverfahren bei Erkrankungen der Lymphbahnen hervorzuheben. Hier ist er einer der wenigen national und international ausgewiesenen Experten. Während seiner Laufbahn wurde er bereits als junger Wissenschaftler mit dem Holthusen-Ring und später mit dem Alfred-Breit-Preis der Deutschen Röntgengesellschaft für seine Verdienste um die interventionelle Radiologie ausgezeichnet.

Als Direktor der Radiologischen Klinik hat er über viele Jahre mit großer Weitsicht alle innovativen Teilbereiche von Radiologie und Strahlentherapie mit großem Erfolg gefördert. Hier sind insbesondere die Mamma-MRT, die MRT bei Patienten mit Herzschrittmacher sowie die KardioMRT und sein frühes Engagement in der interventionellen Onkologie mittels SIRT zu nennen. Mit Einführung der Tomo-Therapie gehörte seine Klinik zudem zu einer der ersten Strahlenkliniken in Deutschland, die diese Methode einer hoch-präzise gesteuerten Bestrahlung in der Krebstherapie anbieten konnte.

Dabei hat Professor Schild immer auch den interdisziplinären Kontext gesucht und bereits sehr früh in der eigenen Klinik Arbeitsgruppen mit Naturwissenschaftlern und Ingenieuren etabliert. Neben dem Einsatz und der Weiterentwicklung von neuesten MR-Techniken wurden dort insbesondere die Verfahren der funktionellen MRT, der MR-Spektroskopie sowie vielfältige Ansätze der quantitativen MRT bearbeitet und schließlich in den klinischen Einsatz überführt.

In seiner Klinik hat Prof. Schild bei seinem außerordentlichen Engagement für For-

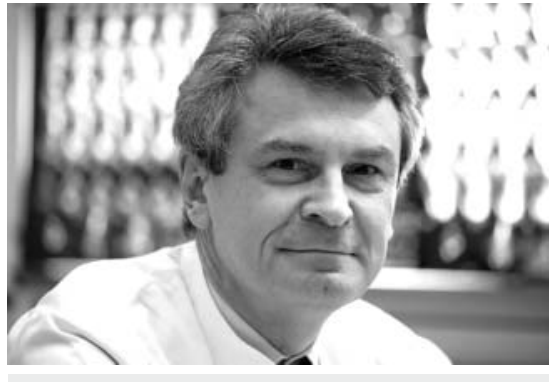

Professor Dr. med. Hans Heinz Schild

schung und Lehre stets großen Wert auf die klinische Ausrichtung der Radiologie und damit auf die Patientenversorgung gelegt. So war sein hoher wissenschaftlicher Anspruch stets an einer Verbesserung von Diagnostik und Therapie und somit am Wohl der Patienten orientiert.

Auszeichnende Merkmale seines Handelns als Vorgesetzter und Klinikleiter waren neben seiner Kontinuität und Verlässlichkeit sein großzügiges Wesen und die Fürsorge für seine Mitarbeiter sowohl in dienstlichen wie auch in persönlichen Belangen. Ganz besonders legte er Wert auf einen kollegialen und freundlichen Umgangston und ein produktives Miteinander aller Mitarbeiter innerhalb seiner Klinik.

Im Namen aller Mitarbeiter der Radiologischen Klinik möchten wir an dieser Stelle die Gelegenheit nutzen und ihm, verbunden mit unseren besten Wünschen, unseren großen Dank aussprechen.

Für die Mitarbeiter der Radiologischen Universitätsklinik Bonn

Daniel Thomas \& Wolfgang Block, Bonn 